\title{
THE DESIGN OF AUTOKINETIC LASER GUN FIRE DEVICE
}

\author{
Lu Xu ${ }^{1, a}$, Enle Gan ${ }^{2, b}$, Shengfei Zheng ${ }^{3, c}$, Feng Wang ${ }^{4, d}$ and Zhiqiang Lu $^{5, \mathrm{e}}$ \\ 1, 2, 3, 4, 5 College of Physics and Electronic Information Engineering Wenzhou University \\ Chashan University Town, Wenzhou 325035, P. R. China \\ d wang_fengwf@163.com, ${ }^{a} 172447327 @ q q . c o m$
}

\begin{abstract}
Keywords: MC9S12XS128 micro controller; STM32F103VE MCU; Two-degrees-of-freedom manipulator; Laser gun; Camera; LCD12864
\end{abstract}

\begin{abstract}
This paper describes an electronic system which can enable the laser gun to aim, shoot and report target automatically. This system consists of a laser gun shoot system based on STM32F103VE MCU and an impact point identification system based on MC9S12XS128 micro controller. Laser gun, placed on a two-degrees-of-freedom manipulator, consists of a laser transmitter telescope, laser transmitter telescope drive circuit and laser modulation tube. Changing the two-degrees-of-freedom manipulator horizontal and vertical angle through the matrix keyboard, STM32F103VE MCU, can control the horizontal and vertical movement of the impact point emitted by the laser gun on a shooting target. Camera recognizes the location of impact point on the shooting target. In addition, LCD12864 displays the ring number, orientation of impact point and the shooting target corresponding graphics.
\end{abstract}

\section{Design content and requirements}

This design is an electronic system which can control laser gun's aiming, shooting and reporting target automatically based on STM32F103VE MCU.

A laser gun emit beams which can be controlled by the circuit. The impact point of the laser gun can be controlled by the matrix keyboard which enables the laser beam to move vertically and horizontally on the shooting target.

\section{Design and Proof}

The system consists of two parts, the laser gun shoot system and the impact point identification system which requires two micro-controllers. MC9S12XS128 processes spot image on the shooting target collected by camera, gets location information and sends the location information by wireless transmission module to STM32103VE MCU. STM32F103VE MCU controls the two-degrees-of-freedom manipulator which enables the laser gun to move in two dimensions on the shooting target. The location information is displayed on the LCD12864 both in words and in images. Key module and display module realize the human-computer interaction function, completing the system requirements.

\subsection{MCU control module}

1) The single chip controller MC9S12XS128 is a powerful 16-bit micro controller with rich on-chip resources. It is a preferred processor taking into considerations low power consumption requirements.

2) STM32F103VE MUC, 32 processors, has a powerful information processing ability. So it can achieve system requirements perfectly and is suitable as an automatic control system controller.

The STM32F103VE has a very strong image information processing capabilities, therefore we choose the STM32F103VE MCU as the main controller execution units, and choose MC9S12XS128 as a vice-controller, being responsible for processing camera images.

\subsection{Motor Selection}

The main task of a motor is making the laser gun rotate, adjusting the location of the impact point on the shooting target, and then controlling the impact point to appear in the specified location 
as required.

1) The two consecutive servo motors drive laser gun. Due to the large rotation angle (in the range of 0-360 degrees), it is not easy to achieve the control of the laser gun two-dimensional movement.

2) The two-degrees-of-freedom manipulator drives head laser gun. The system uses PWM wave which input cycle is $20 \mathrm{~ms}$. Duty cycle $0.5 \mathrm{~ms}$ to $2.5 \mathrm{~ms}$ corresponds to 0 degrees to 180 degrees.

The design of the main motor execution unit requires high accuracy control, and its efficiency is directly related to the completion of this design. It is after comprehensive and continuous considerations that the two-degrees-of-freedom manipulator is chosen eventually in this design.

\subsection{Laser gun, camera and display Module design}

The laser gun equips an optical lens, which makes the laser still bright far away. Black tape is used to keep the laser gun's switch closed all the time.

Camera module uses models of black and white digital camera OV7620. Using OV7620 is very convenient as it can be powered directly through the micro controller. As a result, this module can collect the location of impact point area of laser gun on shooting target by camera which is controlled by MC9S12XS128 single-chip micro computer.

The LCD12864 display is a 128*64 characters dot matrix graphics LCD module. It can display characters and graphics and has good picture images and high resolution. It can interface directly with the CPU, can display the graphics of shooting target and the impact point information perfectly.

\section{Laser guns shoot system hardware design}

\subsection{Laser guns shoot system}

STM32F103VE execution unit, a main controller, has a matrix keyboard input, a wireless receiver, a liquid crystal display, a laser gun and a two-degrees-of-freedom manipulator. It enables the two-degrees-of-freedom manipulator to move in two dimensions and the laser gun to aim and shoot automatically. The partial block diagram is shown in Figures 1 and 2.

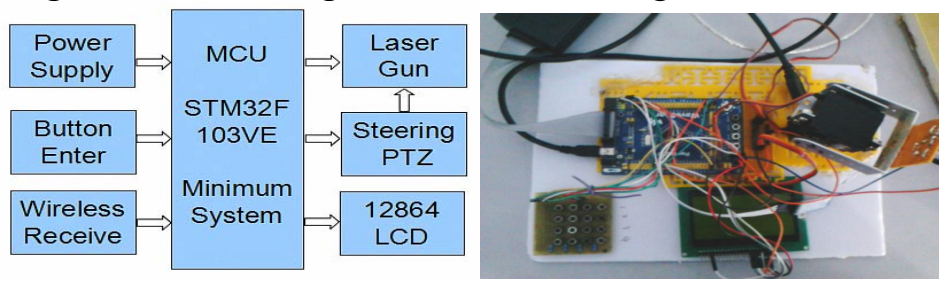

Figure 1. laser gun shoot system charts

Figure 2. laser gun shoot system physical map

\subsubsection{STM32F103VE MCU module}

MCU STM32F103VE's PE1 port connects to laser gun, which makes the laser gun aim and shoot automatically. Pin PD9 and PD10 port connect PWM wave out port, and then connect to two-degrees-of-freedom manipulator control terminal. PD9 controls its horizontal rotation, and PD10 controls its vertical movement; Pin PB5, PB6, PB7 and PB8 connect to the matrix keyboard output pins; Pin PB9, PB10, PB11 and PB12 connect to matrix keyboard input pins. The matrix keyboard controls the laser gun's aiming and shooting at the specified location. In addition, Pin PC1, PC2, PC3, PC4 connect to the LCD's data terminal.

\subsubsection{Keyboard Circuit}

In order to reduce the occupancy of the I/O port, we decided to use $4 \times 4$ matrix keypad. Matrix keyboard is made of row and column lines and the buttons are located on the crossings. When a key is pressed, the intersection of the row and column lines are connected, and the corresponding row or column line's electrical level will change. MCU can determine which key is pressed by detecting electrical level changes in the row or column. PB5, PB6, PB7, PB8 is low outputted by line. PB9, PB10, PB11, PB12 is inputted by column. MCU uses line scan method to discriminate matrix keyboard. The key is pressed as long as the low electrical level appears.

The way to identify the location of the closed key: If a key is pressed, MCU will set the four row line PB5-PB8 low electrical level respectively. For instance when PB5 electrical level is low, 
PB6-PB8 electrical level is high, and then MCU will check the column line PB9-PB12 electrical level one by one to identify which one is low electrical level. If PB9 electrical level is low, the key at the junction of column lines PB9 and row lines PB5 is the closed button.

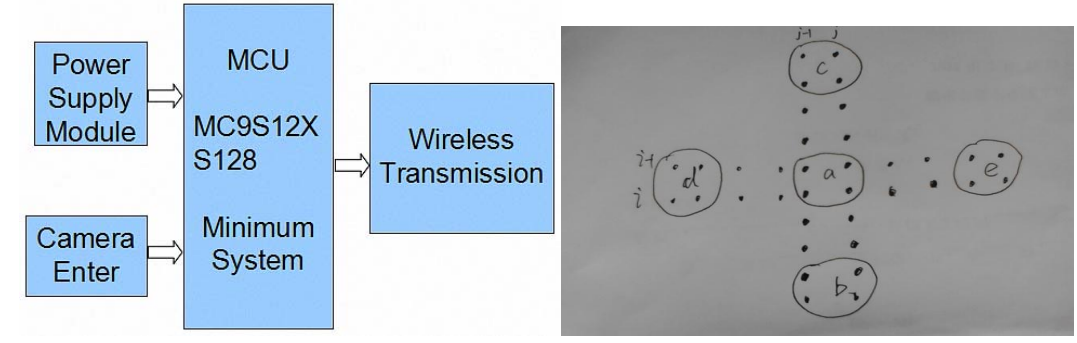

Figure 3. matrix keyboard

\subsection{Impact point recognition system}

Figure 4. impact point recognition system charts

MCU MC9S12XS128, the vice controller, is responsible for processing the data of image collected by camera, displaying position information of the image and connecting to camera and liquid crystal display 12864.

\section{Theoretical analysis and calculation}

\subsection{Identify the impact point}

Identify the location of impact point according to the data from the camera. When the sum gray value of the four pixels in the middle zone is greater than the sum gray values of the four pixels in every ambient zone (left, right, up, down), it is determined that the middle zone is the impact point. Just as shown in Figure 7, take a, b, c, d, e five points respectively, and calculate the sum gray value of these five points.

$a=$ Buffer[i] $[j]+$ Buffer[i][j-1] + Buffer[i-1][j] + Buffer[i-1][j-1];

$b=$ Buffer $[i+4][j]+B u f f e r[i+4][j-1]+B u f f e r[i+3][j]+B u f f e r[i+3][j-1]$;

$c=$ Buffer $[\mathrm{i}-4][\mathrm{j}]+$ Buffer[i-4][j-1]+Buffer[i-5][j] + Buffer[i-5][j-1];

$d=$ Buffer[i] $[j-4]+B u f f e r[i][j-5]+B u f f e r[i-1][j-4]+B u f f e r[i-1][j-5]$;

$\mathrm{e}=$ Buffer[i][j+4]+Buffer[i][j+3]+Buffer[i-1][j+4]+Buffer[i-1][j+3];

Compare which one is the maximum among a, b, c, d, e. The maximum one is the impact point. Take $i, j$ as the impact point's row and column respectively, and then use the wireless module to transmit $\mathrm{i}, \mathrm{j}$ to STM32F103VE MCU. It can be seen S $=\operatorname{sqrt}((\mathrm{Sx}-30) *(\mathrm{Sx}-30)+(\mathrm{Sy}-30) *(\mathrm{Sy}-30))$, through path analysis to calculate the value of $\mathrm{S}$, then MCU can determine the ring number of impact point.

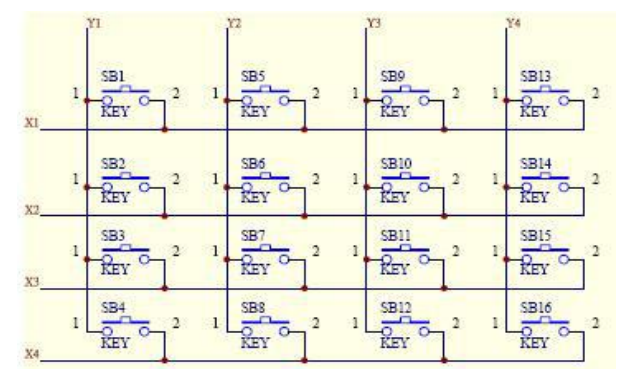

Figure 5. Nonadjacent impact point mode map

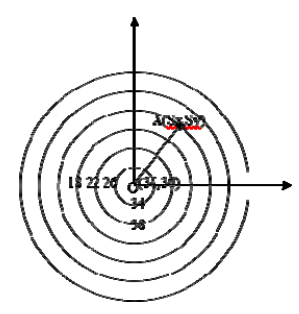

Figure 6. coordinate system

\subsection{Target Accuracy Analysis}

When the PWM duty ratio inputted by STM32F103VE MCU is changing, two-degrees-of-freedom manipulator will enable laser gun to move in two dimensions. The distance of laser head to the bull's-eye is $3 \mathrm{~m}$ actually, the target of the largest radius is $30 \mathrm{~cm}$, the maximum angle of the laser gun is $2 * \arctan (0.3 / 3)=5.677$ degrees, the smallest angle is $\arctan (0.05 / 3)=$ 0.955 degrees. 


\section{System software design}

The program of this system mainly includes image acquisition, processing algorithms and laser gun control. After obtaining image information, this system will turn into different modes to perform different functions.

After making the system initialization, the laser gun's aiming and shooting at the predetermined location is controlled by two-degrees-of-freedom manipulator. The main function program flow chart is shown in figure 7.

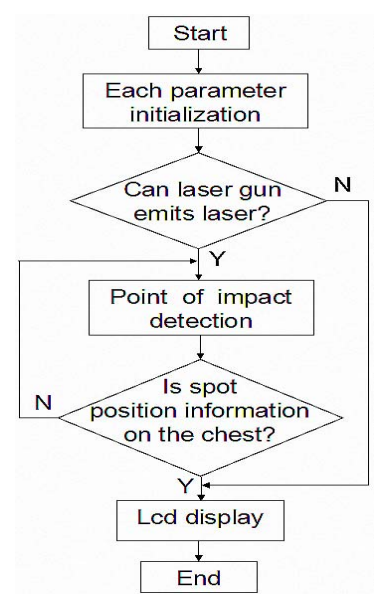

Figure 7. The main program flow chart

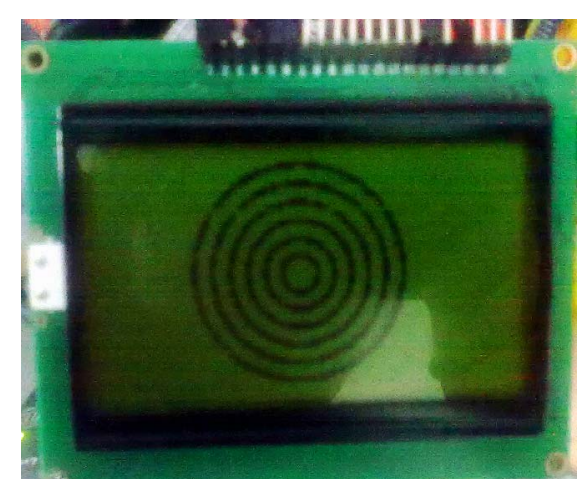

Figure 8. Shooting target image

\section{Results analysis}

The laser gun can achieve directional control. In addition, the impact point spot diameter of the laser beam on the shooting target is less than $5 \mathrm{~mm}$. Laser gun can aim and hit the bull's-eye from the predetermined location on the shooting target quickly within 15 seconds. Laser gun can be controlled to aim and shoot the corresponding position on the shooting target according to random number of ring which is set artificially. When the laser gun shoots at the shooting target, the corresponding graphics of the impact point and shooting target is be displayed on the LCD12864.

\section{Conclusions}

The control systems and motor selection, as well as the two-degrees-of-freedom manipulator control algorithm - which allows to achieve the accuracy of the laser gun movement - play a critical role in the laser gun shoot systems. After three months of research and manufacture, the design has ultimately achieved the requirements. An electronic system which can control the laser gun's aiming, shooting, and reporting target automatically has been successfully designed.

\section{Acknowledgments}

This work is supported by the Opening Laboratory Project of Wenzhou University (14SK26A), the Young Talent Fostering Program of Zhejiang Province (2014R424016), the Innovative and Entrepreneurial Training Project of College Students in Wenzhou University (2013121232), the Wenzhou 551 Project and the Zhejiang Provincial Natural Science Foundation of China (Grant No. Q14F030024). The authors also gratefully acknowledge the helpful comments and suggestions of the reviewers, which have improved the presentation.

\section{References}

[1] Ren Guoguang: Tactical High Energy Laser Weapons Development Status and Future, Laser and infrared, 2002, 32 (4); 211-217. 
[2] Lu Yong-Jun, Qu Yanling, Zhang Ping, Wang Limei, Zheng Jianzhou: New precision shooting laser simulation system design [J] Dalian Nationalities University, 2011,13 (3): 270-273.

[3] Wu Lei, Xieai Zhen, Bian Feng, Meng Zhi: Training new laser targeting system Design and Implementation, Programmable Controller \& Factory Automation, 2011.

[4] Feng Wang, Yangu Zhang: The Comprehensive Defence against Railway System-wide Disaster by Computer Interlocking System, Proc. 2nd Int'l Conf. Intelligent Information Management Systems and Technology (IIMST 2007), World Academic Press, 2007, pp. 95-98.

[5] Feng Wang, Yangu Zhang, and Chengfeng Ye: HBACA-based Railway Yard Route Searching, The 3rd International Symposium on Intelligent Information Technology Application, 2009.11, pp.496-499.

[6] Feng Wang, Bo-lin Wang, and Yangu Zhang: Asymmetry DFT Algorithm and Its Application, Journal of Chongqing University, Vol. 31, No. 2, Feb. 2008, pp.162-165. 